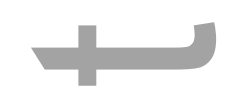

Title of the paper: The Proteomes of the Human Eye, a Highly Compartmentalized Organ

Author's Full Name: Gilbert S. Omenn

Author's Institute Name: University of Michigan

Correspondence:

Dr. Gilbert S. Omenn

Director, Center for Computational Medicine \& Bioinformatics

100 Washtenaw Avenue, Suite 2065B

Ann Arbor, MI 48109-2218, U.S.A.

E-mail: gomenn@umich.edu

Fax: (734) 615-6553

Keywords:

Biology and Disease-driven Human Proteome Project

End Blindness by 2020

Eye proteome

EyeOme

Human Proteome Project

This is the author manuscript accepted for publication and has undergone full peer review but has not been through the copyediting, typesetting, pagination and proofreading process, which may lead to differences between this version and the Version of Record. Please cite this article as doi: 10.1002/pmic.201600340.

This article is protected by copyright. All rights reserved. 
Total number of words (including references and figure legend): 1783
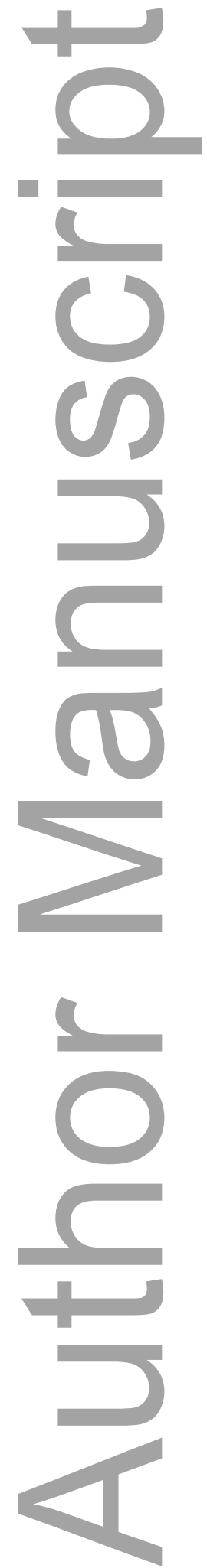

This article is protected by copyright. All rights reserved. 


\section{Abstract Page}

Proteomics has now published a series of Dataset Briefs on the EyeOme from the HUPO Human Proteome Project with high-quality analyses of the proteomes of these compartments of the human eye: retina, iris, ciliary body, retinal pigment epithelium/choroid, retrobulbar optic nerve, and sclera, with 3436, 2929, 2867, 2755, 2711, and 1945 proteins, respectively. These proteomics resources represent a useful starting point for a broad range of research aimed at developing preventive and therapeutic interventions for the various causes of blindness.

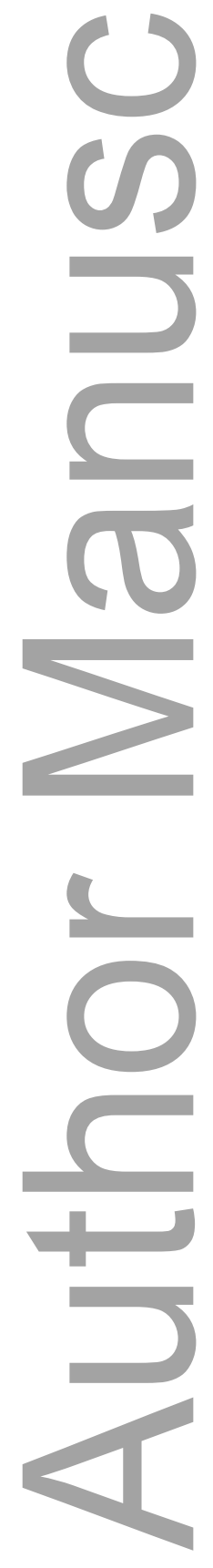

This article is protected by copyright. All rights reserved. 
Proteomics has now published a series of four papers on the human eye proteome [1-4]. Under the aegis of the Human Proteome Organization Biology and Disease-driven Human Proteome Project (HPP), the EyeOme was organized by Semba and colleagues in 2012. The eye is a complex organ with the tear film, conjunctiva, cornea, aqueous humor, iris, ciliary body, lens, vitreous humor, retina, choroid, sclera, and optic nerve (see Figure 1). Certain features are quite unique, including transparency of the cornea, focal abilities of the lens, and light reception by the retina. The aim of the Human EyeOme Project is to identify and quantitate proteins and their isoforms, variants, posttranslational modifications, and interactions that may mediate the many biological processes and diseases or disorders affecting vision.

The first paper [1] was a literature review of 144 articles cataloguing a total of 4842 non-redundant proteins across 7 compartments of the eye as shown in Figure 1. As was noted [5], it was not feasible to assess the quality of the data or estimate numbers of likely false-positives in the heterogeneous studies from which these findings were summarized. Clearly the numbers of proteins identified rose rapidly with the use of advanced MS instruments. The review provides a useful introduction to the many diseases that arise in the various compartments of the eye, for which proteomics could yield protein biomarkers not only in the eye, but more accessibly in the plasma.

The subsequent three papers in the category Dataset Briefs utilized the Orbitrap Elite with stringent quality filters to analyze the sub-proteomes from a carefully-dissected set of five eyes from normal individuals age 51-76. These represent state-of-the art results by compartment, including four parts of the eye lacking any results in Figure 1.

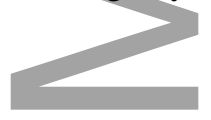

Zhang et al [2] published the retina proteome in February 2015, with 3436 non-redundant proteins, of which $22 \%$ were based on a single peptide, with manual inspection of the spectra and with protein FDR $<1.0 \%$, a big increase over the 672 summarized in the 2013 review. i3D, X!Tandem, and OMSSA search engines were used to match with the UniProt reference database. FDRs were estimated with Peptide/Protein Prophet. They showed the overlap of the 3436 proteins with the 672 previously reported, with the 15058 proteins in the Human PeptideAtlas 2014-07-31, and with 267 predicted proteins from transcripts expressed in retina according to the Human Protein Atlas. Many proteins involved in the rhodopsin-based visual cycle and retinoid metabolism and multiple apolipoproteins were identified. They highlighted 8 novel and 12 known splice isoforms, as well as 158 proteins reported to be associated with age-related macular degeneration and multiple apolipoproteins associated with diabetic retinopathy.

In January 2016, Zhang et al [3] published results for the previously little-studied compartments iris, ciliary body, and retinal pigment epithelium/choroid, with 2959, 2867, and 2755 non-redundant proteins, respectively, at FDR rates of $<0.1 \%$ for peptides and $<1.0 \%$ for proteins (all raw data deposited through ProteomeXchange in PRIDE). Identifications based on single peptides were 19,

This article is protected by copyright. All rights reserved. 
17, and 19\%, respectively. Functional annotations for each proteome were generated and plotted using PANTHER, with rather similar pie charts. Overlaps were shown for these three proteomes with previously published datasets and plasma proteins. There were 43 unambiguous splice isoforms. Only four "missing proteins" (neXtProt PE levels 2-4) were identified by HPP Guidelines v1 with two or more proteotypic peptides. RPE/choroid proteins were also compared with a recently curated list of 189 proteins associated with age-related macular degeneration. As expected, the iris and the ciliary body contained many myosins, tropomyosins, and other proteins related to motor functions, while the $\mathrm{RPE} /$ choroid proteome notably contained enzymes related to visual cycle, retinoid metabolism, complement pathways, and apolipoproteins.

The final paper, by Zhang et al [4] in October 2016, presented the proteomes of normal retrobulbar optic nerve and selera, with 2711 and 1945 non-redundant proteins, 17 and $21 \%$ based on single peptides, and 21 and 10 alternatively spliced isoforms, respectively. These compartments were virgin territory for proteomics. The same stringent FDR thresholds were applied, with the rule of parsimony dictating that only one of a group of proteins to which the identified peptides matched would be counted. For the first time the minimal length for peptides was cited ( $7 \mathrm{aa}$ ). The HPP Guidelines v1 required 7aa; v2.1, used by the HPP for the 2016 special issue of the Journal of Proteome Research [6], required two peptides of at least 9 aa in length (Deutsch et al [7]). Those same requirements have been adopted by neXtProt and PeptideAtlas. As of October 2016, the Human All Build of PeptideAtlas version 2016-01 contains 7400 canonical proteins identified as expressed in the eye [E. Deutsch, personal communication; go to the PeptideAtlas builds selection interface and choose Human Eye to open:

https://db.systemsbiology.net/sbeams/cgi/PeptideAtlas/buildDetails?atlas build id=417].

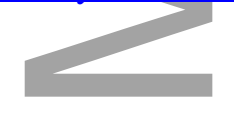

The optic nerve notably contained astrocytic (glial fibrillary acidic protein), oligodendrocytic (laminin, proteolipid protein, fibronectin), and myelin proteins, while the predominant proteins in the sclera were numerous types of collagens plus other extracellular matrix proteins and integrins. Venn diagrams and tables in the Supporting Information provide overlaps between optic nerve, retina, iris, ciliary body, RPE/choroid, sclera, and plasma proteins for the all eye-tissue total of 11,116 proteins. It is a good idea to complement these subproteomes with bibliometric analyses for the top 50 "popular proteins" in each from biological studies, as has been published for six other organ systems (cardiovascular, hepatic, renal, pulmonary, cerebral, and intestinal) by Lam et al [8]).

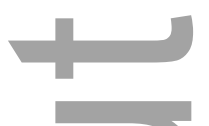

These normal eye proteome resources serve as a foundation for disease-related studies of the eye, both directly in tissue and fluid compartments of the eye and using plasma protein biomarker candidates, especially with SRM targeted proteomics methods and resources [9].

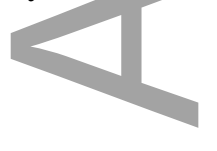

This article is protected by copyright. All rights reserved. 
A prominent entrepreneur and philanthropist, Sanford Greenberg, blind since age 19 years, issued a Challenge to End Blindness by 2020 , with a prize of gold valued at $\$ 3$ million. The award will go to the research team making the most dramatic progress in diagnosis, treatment, or prevention of various causes of blindness. Already there are promising advances using gene therapy, retinal implants, stem cell treatments, and other technologies. Greenberg announced the prize on 18 October 2012 and projected the award period 2978 days to 13 December 2020, the same interval as President Kennedy's 1961 speech about sending a man to the moon and bringing him back safely, which was accomplished in 1969. In 2014 he appeared on the Charlie Rose Show on PBS TV and expanded the original \$2M value to $\$ 3 \mathrm{M}$. Greenberg said, “As President Kennedy believed in our capacity to defy current knowledge and reach the moon, so we believe in the ability of scientists and researchers throughout the world to end blindness. But - a critical "but" - no one in this field has attempted to correct for the same sort of lacks that President Kennedy pinpointed: marshaling the resources ... specifying the long-range goal $\ldots$ a radical refocus on the ultimate objective - to end blindness - is needed to energize and bring together researchers, just as the refocus of the objective advocated by Dr. Jonas Salk led to the effective end of the scourge of polio." The awardee will be chosen by an independent Governing Council [https://endblindnessby2020.com]. This is an opportunity for eye proteomics researchers!

The National Academy of Medicine on 15 September 2016 released a major report about preventing eye diseases [10] which provides current context for planning disease-related proteomics studies of the eye. The Human EyeOme Project is one of 22 teams of the Biology and Disease-driven Human Proteome Project [11].

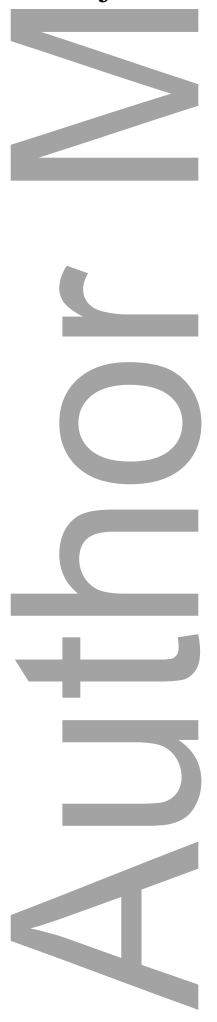

This article is protected by copyright. All rights reserved. 
Figure 1 Legend: Schematic diagram of the human eye, from Semba et al [1], with the number of non-redundant proteins identified in various tissues and biofluids of the eye as of 2013; no results were available at that time for iris, ciliary body, optic nerve, or sclera, all of which have been analyzed subsequently. As summarized in this Commentary, now retina, iris, ciliary body, retinal pigment epithelium/choroid, retrobulbar optic nerve, and sclera have 3436, 2929, 2867, 2755, 2711, and 1945 proteins, respectively.
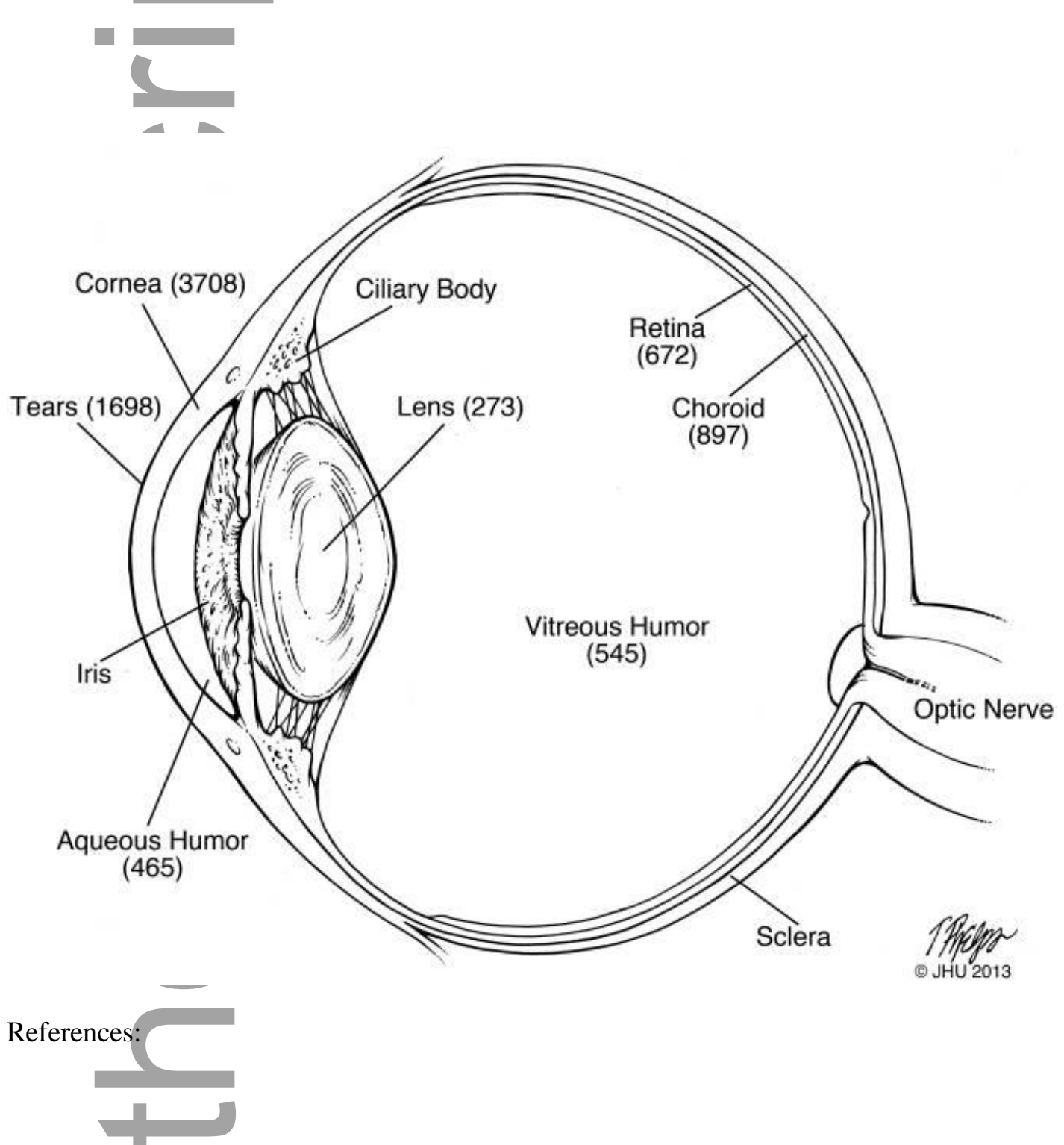

[1] Semba, R. D., Enghild, J. J., Venkatraman, V., Dyrlund, T. F., Van Eyk, J. E., The Human Eye Proteome Project: perspectives on an emerging proteome. Proteomics 2013, 13, 2500-2511.

[2] Zhang, P., Dufresne, C., Turner, R., Ferri, S., et al., The proteome of human retina. Proteomics $2015,15,836-840$.

[3] Zhang, P., Kirby, D., Dufresne, C., Chen, Y., et al., Defining the proteome of human iris, ciliary body, retinal pigment epithelium, and choroid. Proteomics 2016, 16, 1146-1153.

This article is protected by copyright. All rights reserved. 
[4] Zhang, P., Karani, R., Turner, R. L., Dufresne, C., et al., The proteome of normal human retrobulbar optic nerve and sclera. Proteomics 2016, 16, 2592-2596.

[5] Omenn, G. S., The human eye proteome project. Proteomics 2013, 13, 2375-2376.

[6] Paik, Y. K., Overall, C. M., Deutsch, E. W., Hancock, W., Omenn, G. S., Progress in chromosomecentric Human Proteome Project as highlighted in the annual special issue IV. J. Proteome Res. 2016. [In press].

[7] Deutsch, E. W., Overall, C. M., Van Eyk, J., Baker, M., et al., Human Proteome Project mass spectrometry data interpretation guidelines 2.1.2016 Aug 24 [Epub ahead of print]. PMID: 27490519. DOI: 10.102/acs.jproteome.6b00392.

[8] Lam, M., Xing, Y., Lau, E., Cao, Q., et al., Data-driven approach to determine popular proteins for targeted proteomics translation. J. Proteome Res. 2016 Jul 19 [Epub ahead of print]. PMID: 27356587. DOI: 10.102/acs.jproteome.6b00095.

[9] Kusebauch, U., Campbell, D. S., Deutsch, E. W., Chu, C. S., et al., Human SRMAtlas: A Resource of Targeted Assays to Quantify the Complete Human Proteome. Cell 2016, 166, 766-778.

[10] National Academies of Sciences, Engineering, and Medicine, Board on Population Health and Public Health Practice; Health and Medicine Division, Making Eye Health a Population Health Imperative: Vision for Tomorrow, National Academies Press. Washington, D.C. 2016.

[11] Van Eyk, J.E., Corrales, F. J., Aebersold, R., Cerciello, F., et al., Highlights of the Biology and Disease-driven Human Proteome Project, 2015-2016. J. Proteome Res. 2016 Sep 20 [Epub ahead of print]. PMID: 275573249. DOI: 10.1021/acs.jproteome.6b00444.

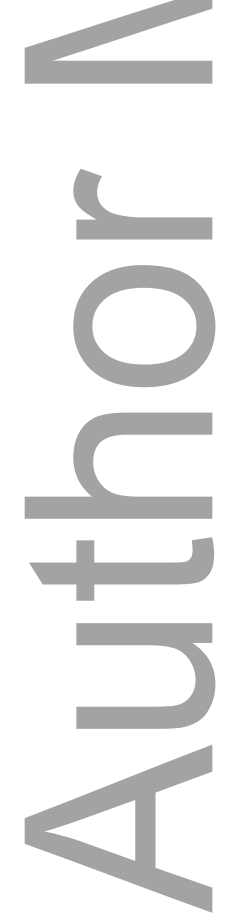

This article is protected by copyright. All rights reserved. 